\title{
Micro-plate magnetic chemiluminescence immunoassay and its applications in carcinoembryonic antigen analysis
}

\author{
LI ZhiYong ${ }^{1}$, ZHANG QianYun ${ }^{2}$, ZHAO LiXia $^{1 *}$, LI ZhenJia $^{3}$, HU GuoMao $^{3}$, \\ LIN Jin-Ming ${ }^{2 *} \&$ WANG Shan ${ }^{4}$ \\ ${ }^{1}$ State Key Laboratory of Environmental Chemistry and Ecotoxicology, Research Center for Eco-Environmental Sciences, \\ Chinese Academy of Sciences, Beijing 100085, China; \\ ${ }^{2}$ Department of Chemistry, Tsinghua University, Beijing 100084, China; \\ ${ }^{3}$ Beijing Chemclin Biotech Co., Ltd., Beijing Academy of Science and Technology, Beijing 100094, China; \\ ${ }^{4}$ School of Chemistry and Chemical Engineering, Xianyang Normal College, Xianyang 712000, China
}

Received May 15, 2009; accepted May 20, 2009

\begin{abstract}
A micro-plate magnetic chemiluminescence immunoassay was developed for rapid and high throughput detection of carcinoembryonic antigens (CEA) in human sera. This method was based on a sandwich immunoreaction of fluorescein isothiocyanate (FITC)-labeled anti-CEA antibodies, CEA antigens, and horseradish peroxidase (HRP)-conjugated anti-CEA antibodies in micro-plate. The immunomagnetic particles coated with anti-FITC antibodies were used as the solid phase for the immunoassay. The separation procedure was carried out by a magnetic plate adaptor and the luminol-hydrogen peroxide $\left(\mathrm{H}_{2} \mathrm{O}_{2}\right)-\mathrm{HRP}$ system was employed for the chemiluminescence detection. The proposed method combined the advantages of the micro-plate reactor and magnetic particle separation technology with the linear range of 5-250 $\mathrm{ng} \mathrm{mL}^{-1}$. The detection limit of CEA was $0.61 \mathrm{ng} \mathrm{mL}$. The coefficient of the variation was less than $7 \%$ and $13 \%$ for intra-assay and inter-assay precision, respectively. Compared with the commercial micro-plate chemiluminescent kit, the proposed method showed a good correlation.
\end{abstract}

micro-plate magnetic chemiluminescence immunoassay, carcinoembryonic antigen, tumor marker

\section{Introduction}

Early diagnoses of cancer play a vital role in clinical therapy, therefore, the measurement of tumor markers becomes one of the most rapidly developing areas in laboratory medicine [1]. Carcinoembryonic antigen (CEA) is a member of the cell surface glycoproteins family with a molecular mass of about $200 \mathrm{kDa}$. It is responsible for clinical diagnosis of many carcinomas such as colon cancer, lung cancer, urothelial carcinoma, ovarian carcinoma, oral cancer and breast cancer [2-5]. It was found that serum from individuals with many carcinomas had higher levels of CEA than

*Corresponding author (email: zlx@ @rcees.ac.cn; jmlin@mail.tsinghua.edu.cn) healthy individuals' serum [6]. Thus, the determination of CEA level was significant in clinical tumor diagnoses.

Immunoassay is usually used for the quantitative determination of CEA, among which radioimmunoassay (RIA) was one of the main methods for CEA detection in the 1980s or even earlier [7-9]. However, RIA method gradually disappeared in recent reports, because it suffered from the potentially harmful effect of the radioactive labels to the operators and the short half-life. Thereafter, enzyme-linked immunosorbent assays (ELISA) [10, 11] and chemiluminescence immunoassay (CLIA) [12, 13] were widely used in clinical test and many commercially available assay kits were developed. Besides, colorimetric immunoassay [14], time-resolved fluoroimmunoassay [15, 16], and liposome immunoassay [17, 18] have also been reported for CEA 
analysis. In recent years, the electrochemical immunosensor has been one of the attractive analytical tools used in CEA detection due to its simplicity and high sensitivity $[19,20]$. However, all of these methods cannot meet the highthroughput disease screening requirements or they are not as available as commercial assay kits.

Due to the requirement of high throughput, high sensitivity and wide linear range in CEA detection, CLIA is becoming one of the most effective methods. Among them, the micro-plate CLIA and the magnetic particle-based CLIA are more commonly used. The application of the micro-plate gives a high flux of analysis and higher sensitivity $[21,22]$. However, the coating process calls for waste of antibodies and brings high requirements of each cell's uniformity and absorption intensity. Moreover, because physical adsorption is the main conjugate force between antibodies and microwells, the limited surface area restricts the linear range of immunoassay. Small super-paramagnetic particles coated with antibodies can disperse evenly into the reaction mixture, affording large surfaces and rapid reaction kinetics, and can also allow large linear range and convenient separation [23]. However, the transparent tube was commonly used as the reaction vessel when using magnetic particles as the solid phase, the transparence difference between the tubes brings in experimental variability and the high non-specific adsorption which reduces the sensitivity.

To solve these deficiencies, we developed the microplate magnetic chemiluminescence immunoassay (MMCLIA) to carry out tumor markers detection work. MMCLIA uses magnetic particles as the solid support and the micro-plate as the reactor, so it easily solves the variation problem caused by the difference between transparent tubes and the ununiformity of the coating conditions among microwells. At the same time, it also solves the problem of coating limitation in micro-plate CLIA method. Furthermore, to avoid the high non-specific absorption in the method based on the magnetic particles, we only need to block with BSA before experiment. Therefore, MMCLIA shows a great research value.

The micro-plate magnetic technologies were rarely reported [24-26] and no report was found in clinical applications. Our group has carried out a series of work on chemiluminescence immunoassay in recent years [27-30], and we have found that MMCLIA shows many advantages such as high throughput, low non-specific adsorption, wide linear range, and low cost. Zhao [31] invented a magnetic separator for the micro-plate and developed a rapid and highthroughput MMCLIA for analysis of $17 \beta$-estradiol in water samples. However, the magnetic separator suffers from the non-homogeneously magnetic field and the difficulty to be automated. In this research, we used a new magnetic separator which was composed by multiple cylindrical magnets positioned in 4 times 6 arrays, and applied MMCLIA to CEA analysis. Through the comparison between this method and commercial micro-plate chemiluminescent kit, we got a good correlation and satisfactory results.

\section{Materials and methods}

\subsection{Apparatus}

The chemiluminescence detection was carried out using a BHP9504 microtiter plate chemiluminescent analyzer (Beijing Hamamatsu Photons Technology, Beijing, China). A DEM-3 automatic micro-plate washer (Tuopu Analytical Instrument, Beijing, China) was employed to blend the solutions in microwells. The incubation procedure at $37{ }^{\circ} \mathrm{C}$ was carried out at an electric heat constant temperature incubator (Chang'an Science Instrument Company, Beijing, China). White 96-well flat-bottomed microplates (InTec Products, Inc. Xiamen, China) were used, and the separation procedure was carried out by a Magnabot 96 magnetic separation device (Promega Biotech, Wisconsin, USA).

\subsection{Chemicals and solutions}

CEA stock solution, HRP-conjugated monoclonal antibodies, Fluorescein isothiocyannate (FITC) and CEA monoclonal antibodies were obtained from Chemclin Biotech (Beijing, China). Chemiluminescent substrates were purchased from DPC (Los Angeles, America). BSA was from Merck (California, USA). Commercial chemiluminescent kit was purchased from Monobind Inc. (California, USA). The immunomagnetic particles $\left(5 \mathrm{mg} \mathrm{mL}^{-1}\right)$ coated with antiFITC antibody and suspended in solution were purchased from Adaltis, Inc. (Roma, Italy).

The plates were post-coated with $300 \mu \mathrm{L} 1 \%(w / v)$ BSA in phosphate buffered saline (PBS) buffer for $12 \mathrm{~h}$ at $4{ }^{\circ} \mathrm{C}$. The washing buffer was phosphate buffer with $0.1 \%(v / v)$ Tween-20.

Normal mixed human sera were obtained from Chemclin Biotech (Beijing, China). Test human sera were obtained from a hospital in Beijing, China, and analyzed without any pretreatment.

\subsection{FITC-labeled anti-CEA antibody}

The FITC labeled anti-CEA antibody was prepared according to refs. [32] and [33] with slight modifications. AntiCEA antibody was put into a dialysis bag and diluted to 1 $\mathrm{mg} \mathrm{mL}{ }^{-1}$, then dialyzed overnight at $4{ }^{\circ} \mathrm{C}$ under stir against $0.2 \mathrm{~mol} \mathrm{~L}^{-1}$ carbonate/bicarbonate buffer at $\mathrm{pH}$ 9.0. FITC was dissolved with a small amount of dimethyl formamide first, and then slowly added into $0.1 \mathrm{~mol} \mathrm{~L}^{-1}$ carbonate/ bicarbonate buffer with stir to obtain the FITC solution $\left(4 \mathrm{mg} \cdot 100 \mathrm{~mL}^{-1}\right)$. The dialyzed anti-CEA antibody was put in FITC solution, and after reaction under stirring for 12-16 $\mathrm{h}$ at $4{ }^{\circ} \mathrm{C}$, the mixture was dialyzed for 3 days at $4{ }^{\circ} \mathrm{C}$ against PBS. The FITC-labeled anti-CEA antibody conju- 
gate was obtained and stored at $-20{ }^{\circ} \mathrm{C}$ for further use.

\subsection{Immunoassay procedure}

2.4.1 Micro-plate magnetic chemiluminescence immunoassay (MMCLIA)

The principle of MMCLIA was shown in Figure 1. First, $35 \mu \mathrm{L}$ CEA standard solutions, $50 \mu \mathrm{L}$ HRP-labeled antiCEA antibody and $50 \mu \mathrm{L}$ FITC-labeled anti-CEA antibody were added into the microwell. After incubated for $1.5 \mathrm{~h}$ at $37{ }^{\circ} \mathrm{C}, 25 \mu \mathrm{L}$ immunomagnetic microparticles were added to react with immunoassay reagents for another $10 \mathrm{~min}$ at $37{ }^{\circ} \mathrm{C}$, and the separation procedure was carried out subsequently. Finally, $100 \mu \mathrm{L}$ chemiluminescence substrate solution was added and the signal detection was carried out after incubation for $5 \mathrm{~min}$ at $37^{\circ} \mathrm{C}$.

\subsubsection{Micro-plate chemiluminescence enzyme immunoas-} say (micro-plate CLIA)

The immunoassay procedure was carried out according to the product instruction. Briefly, $25 \mu \mathrm{L}$ CEA standard solutions or serum samples and $50 \mu \mathrm{L}$ HRP-labeled anti-CEA antibody were added into the microwell. After incubated at room temperature for $45 \mathrm{~min}$, five washings were performed. After the washing procedure, $100 \mu \mathrm{L}$ of CL substrate was added. Then the emitted photons were measured by a BHP9504 microtiter reader within 5 to $30 \mathrm{~min}$.

\subsection{Separation method of MMCLIA}

For MMCLIA system, the most important procedure was solid-liquid separation. In this research, a Magnabot 96 magnetic separation device was used to carry out the separation work. This magnetic separation device consists of 24 mini cylinders. When the magnetic separator was applied to the 96-well plate, around every four microwells there was one mini magnetic cylinder, and the immunomagnetic particles in each well were separated on the side wall. This separation showed an obvious advantage because the unwanted solution could be separated by pipette, so it could realize automatic separation. After adding $250 \mu \mathrm{L}$ washing solution, the remaining magnetic particles were suspended again.

\subsection{Cross-reactivity studies}

The cross-reactivity of the CEA was evaluated by using several tumor markers including alpha-fetoprotein (AFP), prostate specific antigen (PSA), carbohydrate antigen 50 (CA50), CA199, and CA153. Cross-reactivity (CR) was calculated as follows: $C R=100 \times C_{(\mathrm{CEA})} / C_{\text {(cross-reactant) }}$, where $C_{\text {(CEA) }}$ refers to the CEA concentration determined by applying the tested cross reactant signal to the dose-response curve, and $C_{\text {(cross-reactant) }}$ refers to the cross-reactant concentration.

\subsection{Data analysis}

The standard and samples were measured by paralleling two wells, and CL intensity values were integrated. Standard curves were obtained by plotting the logarithm of CL intensity (in relative light units, RLUs) against the logarithm of analyte concentration and fitting to a linear equation.

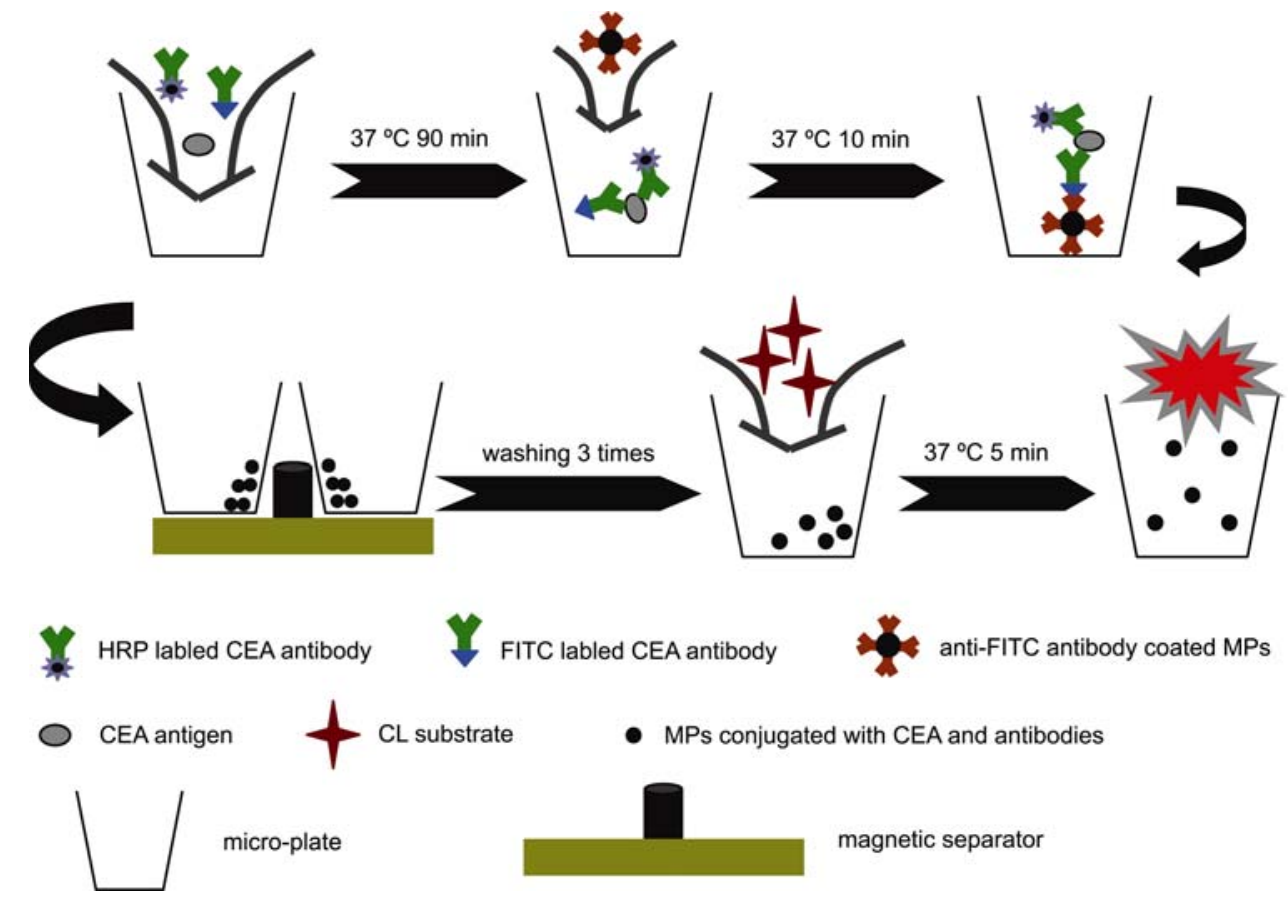

Figure 1 Schematic illustration of the proposed chemiluminescence immunoassay. 


\section{Results and discussion}

\subsection{Influence and optimization of immunoassay re- agents}

As a key parameter affecting the sensitivity and accuracy of immunoassay, the concentration of the HRP-labeled antiCEA antibody and FITC-labeled anti-CEA antibody were optimized. As shown in Figure 2, with the standard CEA concentration of $25 \mathrm{ng} \mathrm{mL}^{-1}$ and the HRP-labeled anti-CEA antibody stock solution concentration of $0.33 \mathrm{mg} \mathrm{mL}^{-1}$, when the dilution ratios of the HRP-labeled anti-CEA antibody were increased from 1:350 to 1:3200, the RLUs also decreased at all concentrations of the examined FITClabeled anti-CEA antibody. Thus, considering both the sensitivity and the assay cost, we selected the 1:350 dilution ratio of the HRP-labeled anti-CEA antibody for further study. As to the concentration of the FITC-labeled anti-CEA antibody, the RLUs were approximately a plateau with the increase of concentration of FITC-labeled anti-CEA antibody from 0.5 to $2 \mu \mathrm{g} \mathrm{mL}^{-1}$, thus we made a further optimization toward the four concentrations in detail (Table 1). FITClabeled anti-CEA antibody concentration of $0.5 \mu \mathrm{g} \mathrm{mL} \mathrm{m}^{-1}$ showed a narrower linear range (the lower $\mathrm{RLU}_{\mathrm{S} 5} / \mathrm{RLU}_{\mathrm{S} 1}$ value), while the other concentrations showed the relatively same sensitivity $\left(\mathrm{RLU}_{\mathrm{S} 1} / \mathrm{RLU}_{\mathrm{S} 0}\right.$ value) and linear range. Therefore, the concentration of $1.0 \mu \mathrm{g} \mathrm{mL}^{-1}$ was selected for our further study.

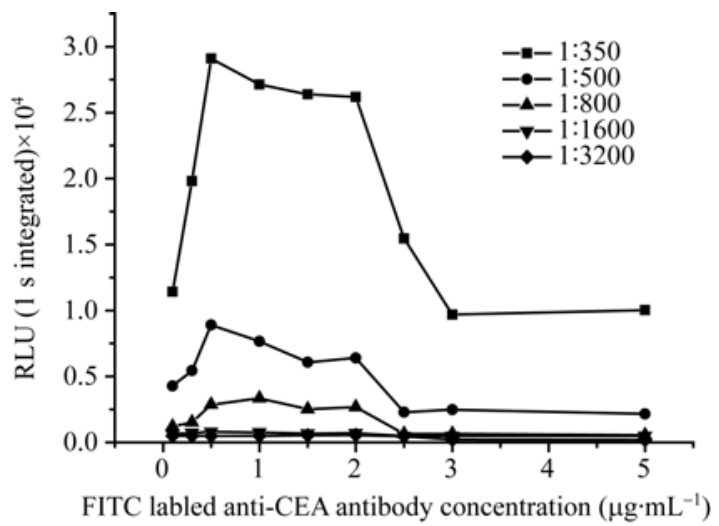

Figure 2 Influence of the dilution ratios of the HRP-labeled anti-CEA antibody and the concentrations of the FITC-labeled anti-CEA antibody, using the standard CEA concentration of $25 \mathrm{ng} \mathrm{mL}^{-1}$. The five curves correspond to a series of dilution ratios of the HRP-labeled anti-CEA antibody (i.e., 1:350, 1:500, 1:800, 1:1600 and 1:3200). Detection conditions: room temperature, $25 \mu \mathrm{L}$ of anti-FITC antibody coated MPs, $100 \mu \mathrm{L}$ of CL substrate.

Table 1 Effect of FITC-CEA concentrations in the presence of HRPCEA with dilution ratio of $1: 350(n=5)$

\begin{tabular}{ccccc}
\hline $\begin{array}{c}\text { FITC-CEA concentration } \\
\left(\mu \mathrm{g} \cdot \mathrm{mL}^{-1}\right)\end{array}$ & 0.5 & 1.0 & 1.5 & 2.0 \\
\hline $\mathrm{RLU}_{\mathrm{S} 1} / \mathrm{RLU}_{\mathrm{S} 0}$ & 2.6 & 3.2 & 3.3 & 3.2 \\
$\mathrm{RLU}_{\mathrm{S} 5} / \mathrm{RLU}_{\mathrm{S} 1}$ & 505.1 & 663.5 & 651.1 & 623.2 \\
\hline
\end{tabular}

3.2 Influence and optimization of physicochemical parameters

\subsubsection{Immunoreaction time and sensitivity}

Immunoreaction time has a direct effect on the sensitivity of the immunoassay. Immunoreaction time from 10 to $180 \mathrm{~min}$ was examined and the results were shown in Figure 3(a). RLUs and sensitivity $\left(\mathrm{RLU}_{\mathrm{S} 1} / \mathrm{RLU}_{\mathrm{S} 0}\right)$ increased as the immunoreaction time increased, because the amount of immunocomplex increased. After $90 \mathrm{~min}$ the value tended to be a plateau, showing the reaction between the antigen and the antibody reached equilibrium. Considering both the assay time and the assay sensitivity, we selected $90 \mathrm{~min}$ as the immunoreaction time.

\subsubsection{Influence of the magnetic particles concentration}

The concentration of the magnetic particles could dramatically influence the analyzing performance. The excessive amount of magnetic particles might lead to the lower sensitivity, because the black magnetic particles could absorb the emitted light. Here, we used a higher antigen concentration $\left(250 \mathrm{ng} \cdot \mathrm{mL}^{-1}\right)$ to evaluate the effect of magnetic particles' concentrations, because the optimized result of magnetic particles under low CEA concentrations could not reflect the linear range appropriately. As shown in Figure 3(b), when the magnetic particles' concentration was $2 \mathrm{mg} \mathrm{mL}^{-1}$, the RLUs reached the highest value. Therefore we set the concentration of magnetic particles to be $2 \mathrm{mg} \mathrm{mL}^{-1}$ as the optimal condition.

\subsubsection{Influence of the volume of the substrate}

The volume of the chemiluminescent substrate was also an important factor. We have examined the substrate volume from 50 to $150 \mu \mathrm{L}$, shown in Figure 3(c), and found RLUs increasing with chemiluminescent substrate volume increased to $100 \mu \mathrm{L}$. After that, decreased RLUs were observed with increasing chemiluminescent substrate volume. This might be caused by the inner filtration effect of the excessive liquid in the microwell. Hence, $100 \mu \mathrm{L}$ of the chemiluminescent substrate was selected.

\subsubsection{Influence of the chemiluminescence reaction time and temperature}

In the chemiluminescence detection procedure, the chemiluminescence incubation time between the chemiluminescent substrate and HRP labeled immunocomplex had a significant influence on the RLUs and the whole chemiluminescence immunoassay sensitivity. The chemiluminescence reaction time and temperature were examined carefully. The result indicated RLUs can be enhanced more quickly when the CL substrate was incubated at $37{ }^{\circ} \mathrm{C}$ than at room temperature. It also showed that the kinetic curves were different between the high HRP concentration and low HRP concentration, which was probably because high HRP concentration could catalyze the reaction between luminal and $\mathrm{H}_{2} \mathrm{O}_{2}$ more quickly. However, whether with high concentra- 

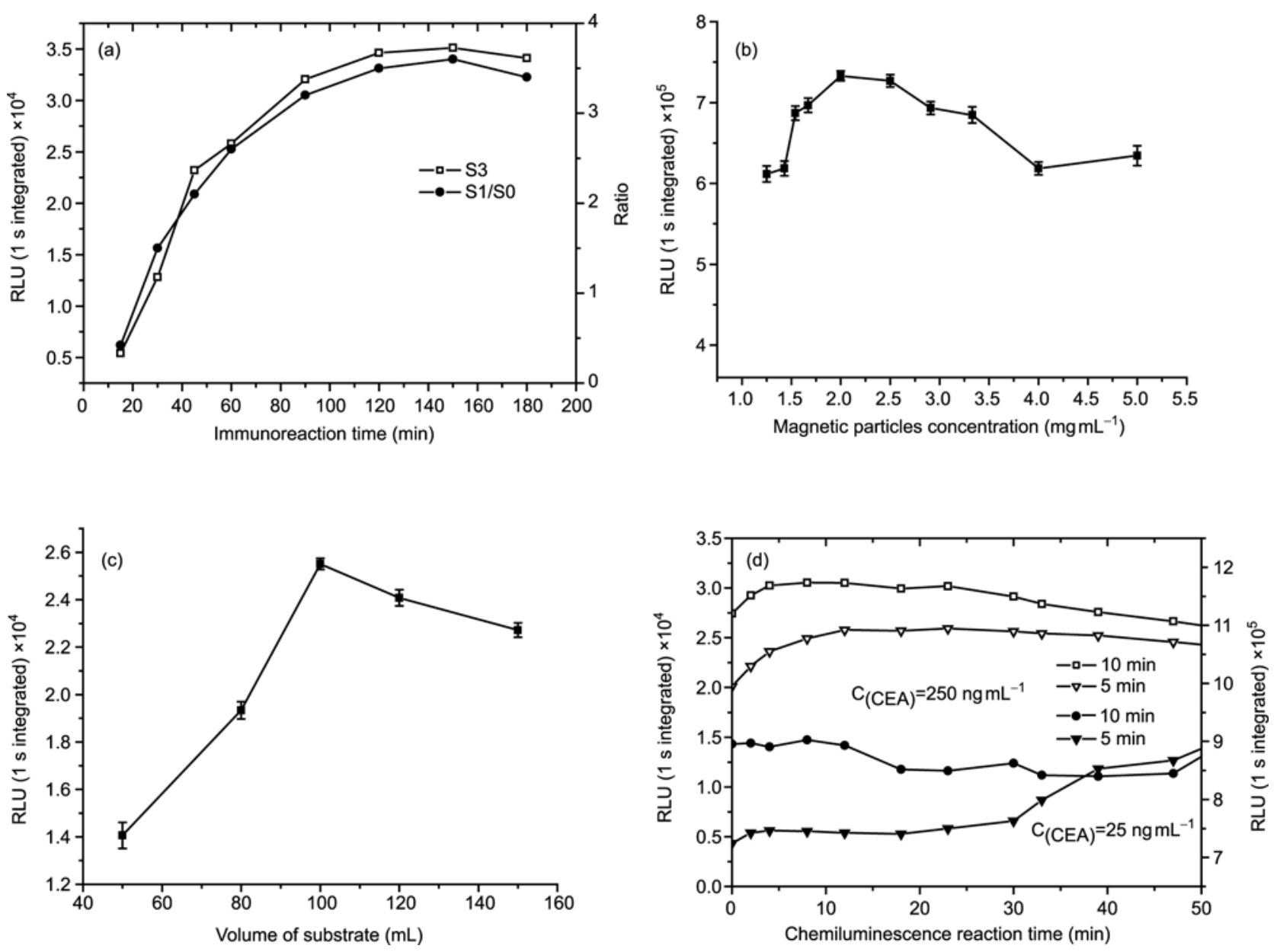

Figure 3 Influence and optimization of the physicochemical parameters. (a) Influence of immunoreaction time, using the standard CEA concentration of $25 \mathrm{ng} \mathrm{mL}^{-1}$. Detection conditions: room temperature, $25 \mu \mathrm{L}$ of anti-FITC antibody coated MPs, $100 \mu \mathrm{L}$ of CL substrate; (b) influence of concentration of MPs, using the standard CEA concentration of $250 \mathrm{ng} \mathrm{mL}^{-1}$. Detection conditions: room temperature, $25 \mu \mathrm{L}$ of anti-FITC antibody coated MPs, $100 \mu \mathrm{L}$ of CL substrate; (c) influence of the volume of the chemiluminescent substrate, using the standard CEA concentration of $25 \mathrm{ng} \mathrm{mL}^{-1}$; (d) influence of chemiluminescence reaction time and temperature. The four curves denote the variation of RLUs upon two antigen concentrations of 25 and $250 \mathrm{ng} \mathrm{mL}^{-1}$, respectively and relevant different reaction time was 5 and $10 \mathrm{~min}$. Detection conditions: $25 \mu \mathrm{L}$ of anti-FITC antibody coated MPs, $100 \mu \mathrm{L}$ of CL substrate.

tion of HRP or low concentration of HRP, the CL intensity could both reach a plateau lasting about $30 \mathrm{~min}$ (Figure 3(d)), after incubation for 5 to $10 \mathrm{~min}$. Thus, chemiluminescence reaction time of $5 \mathrm{~min}$ at $37^{\circ} \mathrm{C}$ was chosen.

\subsection{Non-specific adsorption}

The 96-well micro-plates made of polystyrene showed high non-specific adsorption affinity to proteins. The effect of non-specific adsorption of micro-plate on MMCLIA method was studied. Here, 1\% BSA solution was used for the blocking of the adsorption before the micro-plate was used. As shown in Table 2, the CL intensity almost did not change as the CEA concentration increased when the magnetic particles were absent. After adding the magnetic particles, the non-specific adsorption slightly increased, whereas the RLU intensity increased evidently following the increase of the CEA concentrations.
Table 2 Studies of non-specific adsorption

\begin{tabular}{ccc}
\hline $\begin{array}{c}\text { CEA concentration } \\
\left(\mathrm{ng} \mathrm{mL}^{-1}\right)\end{array}$ & $\begin{array}{c}\text { CL intensity with } \\
\text { magnetic particles absent }\end{array}$ & $\begin{array}{c}\text { CL intensity with } \\
\text { magnetic particles added }\end{array}$ \\
\hline 0 & 331 & 461 \\
5 & 354 & 1551 \\
15 & 329 & 9116 \\
25 & 373 & 23650 \\
100 & 308 & 208000 \\
250 & 362 & 712012 \\
\hline
\end{tabular}

\subsection{Method evaluation}

\subsubsection{Calibration and sensitivity}

Under the optimal conditions, dose-response curve was plotted with the MMCLIA method. The linear range was obtained from 5 to $250 \mathrm{ng} \mathrm{mL}^{-1}$. The detection limit was calculated by taking the mean RLUs signal $\left(\mathrm{S}_{0}, 10\right.$ repli- 
cates) at zero concentration plus two times the standard deviation $\left(\mathrm{S}_{0}+2 \mathrm{SD}\right)$ into the dose-response curve, and the obtained CEA detection limit was $0.61 \mathrm{ng} \mathrm{mL}^{-1}$.

\subsubsection{Precision}

The precision had a close relation to the accuracy and repeatability of the MMCLIA method. Therefore, we studied the details of intra-assay and inter-assay variation using two different concentrations of quality control serum (one is high while the other is low). The intra-assay variation was evaluated by analyzing each concentration ten times per run in 1 day. Similarly, these samples were analyzed on different days using the same protocol ( 2 replicates per run) to obtain the inter-assay variation. The results (Table 3) showed that intra-assay and inter-assay CVs were $<7 \%$ and $<13 \%$, respectively.

\subsubsection{Accuracy}

The accuracy was studied through a recovery experiment with the MMCLIA method. Different amounts of CEA were spiked to the mixed normal human serum to obtain three different distribution involving high, middle and low concentrations. This experiment was repeated five times, and each time there were 5 replicates. The results were shown in Table 4. The average recoveries were between $90 \%$ and $110 \%$, indicating satisfying result of the proposed method.

\subsubsection{Linearity-dilution effect}

The linearity-dilution effect was studied by selecting a certain human serum sample with relatively high concentration. This serum sample was then diluted to a series of concentrations with Calibrator matrix (bovine serum). The results were shown in Figure 4. The relationship between the concentration of diluted CEA and the dilution ratios gave a good linearity correlation coefficient of 0.9986 .

This suggested that the proposed method could be commendably used in the determination of CEA in human serum, and bovine serum could be used to dilute humans' serum before analysis.

\subsubsection{Specificity}

The specificity of the proposed MMCLIA was evaluated using several tumor markers which all have some structures similar to CEA, and their test concentrations were all at least 10 times higher than their normal concentrations in human body. As shown in Table 5, the used CEA antibodies did not cross-react with AFP and PSA, and the CR\% of the other tumor markers were all lower than $3 \%$, which was an acceptable result.

\subsection{Sample analysis and comparison with other methods}

The proposed method was applied to evaluate CEA in human serum. As can be seen in Figure 5, the proposed MMCLIA was compared with the commercial micro-plate chemiluminescent kit and the good agreement was obtained, indicating that the developed MMCLIA could be satisfactorily employed in clinical screening.

\section{Conclusions}

A MMCLIA with high sensitivity, specificity, rapidity, and reproducibility was proposed for the evaluation of CEA in human serum. This research first applied MMCLIA to the determination of tumor marker (CEA) in human serum, and the results correlated well with the commercial micro-plate chemiluminescent kit. The proposed assay combined the micro-plate reactor and magnetic particle separation technology, and made comparisons between the micro-plate CLIA [34] and traditional magnetic particle-based CLIA [35]. At the same time, it also had more advantages: (a) 96well micro-plate was used as the reaction container, realizing high flux work and avoiding the variation caused by the difference among transparent tubes; (b) magnetic particles were used as separation reagent, easily solving the problem

Table 3 Inter-assay and intra-assay variability for CEA

\begin{tabular}{|c|c|c|c|c|c|c|}
\hline \multirow[t]{2}{*}{ Samples } & \multicolumn{3}{|c|}{ Intra-assay } & \multicolumn{3}{|c|}{ Inter-assay } \\
\hline & times of replication & average concentration & $\mathrm{CV}(\%)$ & times of replication & average concentration & $\mathrm{CV}(\%)$ \\
\hline QC1 & 10 & 15.1 & 6.8 & 10 & 14.8 & 11.2 \\
\hline QC2 & 10 & 50.6 & 5.5 & 10 & 51.4 & 12.6 \\
\hline
\end{tabular}

Table 4 Accuracy of the MMCLIA for determination of CEA

\begin{tabular}{|c|c|c|c|c|c|c|}
\hline \multirow{2}{*}{$\begin{array}{l}\text { Spiked amount } \\
\quad\left(\mathrm{ng} \mathrm{mL}^{-1}\right)\end{array}$} & \multicolumn{6}{|c|}{ Recovery (\%) } \\
\hline & $\mathrm{N}_{1}(n=5)$ & $\mathrm{N}_{2}(n=5)$ & $\mathrm{N}_{3}(n=5)$ & $\mathrm{N}_{4}(n=5)$ & $\mathrm{N}_{5}(n=5)$ & average \\
\hline 12 & 104.1 & 111.2 & 98.7 & 106.1 & 101.3 & 104.3 \\
\hline 40 & 102.4 & 105.2 & 99.3 & 103.6 & 99.1 & 101.9 \\
\hline 120 & 89.6 & 92.8 & 101.6 & 95.3 & 94.4 & 94.7 \\
\hline
\end{tabular}




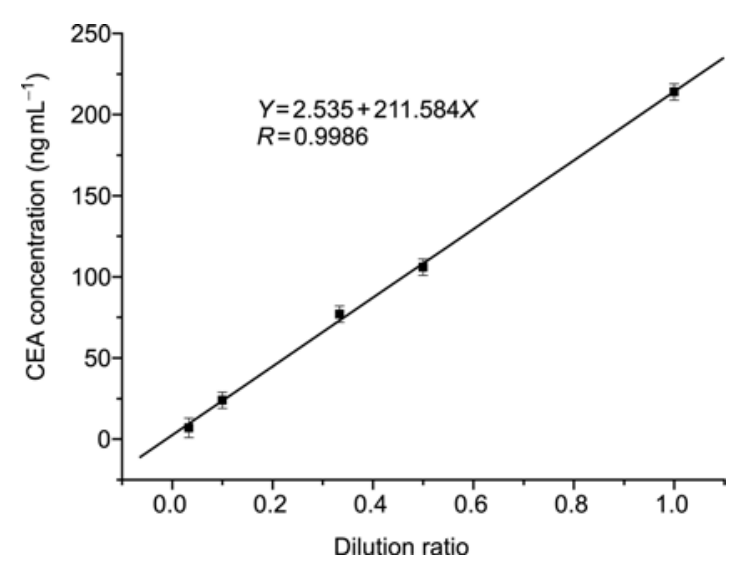

Figure 4 Linearity-dilution effect.

Table 5 Cross-reactivity (CR) of cross-reactants and related compounds

\begin{tabular}{cccc}
\hline Cross-reactants & $\begin{array}{c}\text { Tested } \\
\text { concentration }\end{array}$ & $\begin{array}{c}\text { CEA concentration } \\
\text { determined }\left(\mathrm{ng} \mathrm{mL}^{-1}\right)\end{array}$ & $\mathrm{CR} \%$ \\
\hline AFP & $500 \mathrm{ng} \mathrm{mL}^{-1}$ & $\mathrm{NA}^{\mathrm{a})}$ & 0 \\
PSA & $100 \mathrm{ng} \mathrm{mL}^{-1}$ & $\mathrm{NA}^{\mathrm{a})}$ & 0 \\
CA50 & $140 \mathrm{U} \mathrm{mL}^{-1}$ & 3.03 & 2.2 \\
CA153 & $500 \mathrm{U} \mathrm{mL}^{-1}$ & 5.07 & 1.0 \\
CA199 & $200 \mathrm{U} \mathrm{mL}^{-1}$ & 4.25 & 2.1 \\
\hline
\end{tabular}

a) Not detected.

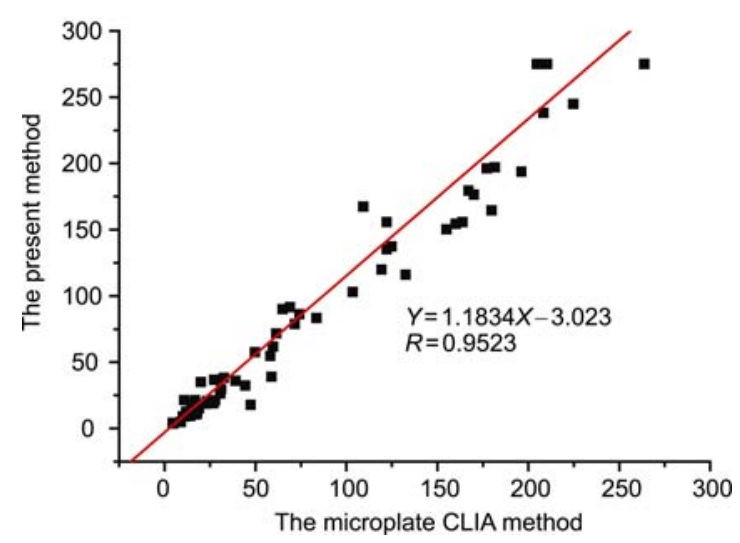

Figure 5 Correlation between results measured by the proposed MMCLIA and the commercial kit.

of ununiformity and quantity limitation in the coating process of micro-plate CLIA, thus it could get much wider linear range [28]; (c) it could reduce the requirements to reaction containers and the amount of reaction reagents, saving cost, and it was more practicable; (d) the new magnetic separation method could realize automatic separation, showing good clinical application perspective and great commercial potential.

The authors express their great thanks for the support from the CAS Major Scientific Research and Equipment Development Projects (YZ-0632), the
National Natural Science Founolation of China (Grant No. 20907060), the CAS Graduate Innovation Foundation (No. YXLW-3), the National key Technology R\&D Program (Grant No. 2006BAF07B03-1-1) and Shaanxi Province, China's Scholarship Council (Grant No. 08JK482)

1 Michael JD. Role of tumor markers in patients with solid cancers: A critical review. Eur J Intern Med, 2007, 18: 175-184

2 Moro D, Villemain D, Vuillez JP, Delord CA, Brambilla C. CEA, CYFRA21-1 and SCC in non-small cell lung cancer. Lung Cancer, 1995, 13: 169-176

3 Kazuya K, Yoshihiro K, Tsunekazu K. Preoperative determination of several serum tumor markers in patients with primary epithelial ovarian carcinoma. Gynecol Obstet Invest, 1999, 47: 52-57

4 Eppler E, Horig H, Kaufman HL, Groscurth P, Filgueira L. Carcinoembryonic antigen (CEA) presentation and specific $\mathrm{T}$ cell-priming by human dendritic cells transfected with CEA-mRNA. Eur J Cancer, 2002, 38: 184-193

5 Carneiro C, Costa L, Melo M, Quintela A, Miranda I, Jorge M, Pina F, Gouveia A, Miguel MJ, Costa EB. Serum tumor markers in metastatic breast cancer comparative study between CEA, CA 15-3 and MCA. Eur J Cancer, 1998, 34: s43

6 Duffy MJ. Carcinoembryonic antigen as a marker for colorectal cancer: Is it clinically useful? Clin Chem, 2001, 47: 624-630

7 Zimmerman R. Improved performance of a double antibody radioimmunoassay for carcinoembryonic antigen. J Immunol Methods, 1979, 25: 311-321

8 Matsuoka YJ, Kuroki M, Koga Y, Ogawa H, Nakazawa N, Tachibana SJ, Minamizawa TK. A new direct solid-phase radioimmunoassay for carcinoembryonic antigen without pretreatment of serum samples. J Immunol Methods, 1983, 58: 31-47

9 Roland VJ, Stein KE. Comparison of RIA and IRMA methods for measurement of carcinoembryonic antigen (CEA). Clin Biochem, 1982, 15: 241-247

10 Mekler VM, Bystryak SM. Electrochemical sensor for immunoassay of carcinoembryonic antigen based on thionine monolayer modified gold electrode. Anal Chim Acta, 1992, 264: 359-363

11 Zhao L, Xu S, Fjaertoft G, Pauksen K. CD66b regulates adhesion and activation of human eosinophils. J Immunol Methods, 2004, 293: 207-214

12 Findeisen R, Albrecht S, Richter B, Deutschmann K, Zimmermann T, Distler W. Chemiluminometric determination of tissue polypeptide antigen (TPA), cancer antigen 15-3 (CA 15-3), carcinoembryonic antigen (CEA) in comparison with vascular endothelial growth factor (VEGF) in follow-up of breast cancer. Luminescence, 2000, 15: 283-289

13 Zhuang HS, Huang JL, Chen GN. Development of a sensitive micro-magnetic chemiluminescence enzyme immunoassay for the determination of carcinoembryonic antigen. Anal Chim Acta, 2004, 512: 347-352

14 Eckert K, Fuhrmann-Selter T, Maurer HR, Buttner P. A colorimetric immunoassay for the detection of E-cadherin and carcinoembryonic antigen, CEA expression on human colon carcinoma cell lines in vitro. Cancer Letters, 1996, 105: 1-4

15 Mastsumoto K, Yuan J, Wang G, Kimura H. Comparison between time-resolved fluorescence immunoassay and electro-chemiluminescence immunoassay for detection of serum carcinoembryonic antigen in colorectal carcinoma. Anal Biochem, 1999, 276: 81-87

16 Yan F, Zhou J, Lin J, Ju H, Hu X. Flow injection immunoassay for carcinoembryonic antigen combined with time-resolved fluorometric detection. J Immunol Methods, 2005, 305: 120-127

17 Ishimori YS, Rokugawa KJ. High sensitive microcapsule immunoassay for protein antigens or antibodies. Anal Chim Acta, 1993, 284: 227-234

18 Haga M, Hoshino S, Okada H, Hazemoto N, Kato Y, Suzuki Y. An improved chemiluminescence-based liposome immunoassay involving apoenzyme. Chem Pharm Bull, 1990, 38: 252-254

19 Dai Z, Chen J, Yan F, Ju HX. Electrochemical sensor for immunoassay of carcinoembryonic antigen based on thionine monolayer modi- 
fied gold electrode. Cancer Detect Prev, 2005, 29: 233-240

20 He XL, Yuan R, Chai YQ, Shi YT. A sensitive amperometric immunosensor for carcinoembryonic antigen detection with porous nanogold film and nano-Au/chitosan composite as immobilization matrix. J Biochem Biophys Methods, 2008, 70: 823-829

21 Zhao L, Lin JM, Li Z, Ying X. Development of a highly sensitive, second antibody format chemiluminescence enzyme immunoassay for the determination of $17 \beta$-estradiol in wastewater. Anal Chim Acta, 2006, 558: 290-295

22 Schneider C, Scholer HF, Schneider RJ. Direct sub-ppt detection of the endocrine disruptor ethynylestradiol in water with a chemiluminescence enzyme-linked immunosorbent assay. Anal Chim Acta, 2005, 551: 92-97

23 Olsvik O, Popovic T, Skjerve E, Cudjoe KS, Hornes E, Ugelstad J, Uhlén M. Magmetic separation techniques in diagnostic microbiology. Clin Microbiol Rev, 1994, 7: 43-54

24 Gundersen SG, Haagensen I, Jonassen TO, Figenschau KJ, de Jonge N, Deelder AM. Magnetic bead antigen capture enzyme-linked immunoassay in microtitre trays for rapid detection of schistosomal circulating anodic antigen. J Immunol Methods, 1992, 148: 1-8

25 Cudjoe KS, Hagtvedt T, Dainty R. Immunomagnetic separation of Salmonella from foods and their detection using immunomagnetic particle, IMP-ELISA. Int J Food Microbiol, 1995, 27: 11-25

$26 \mathrm{Yu} \mathrm{H}$, Ahmed H, Vasta GR. Development of a magnetic microplate chemifluorimmunoassay for rapid detection of bacteria and toxin in blood. Anal Biochem, 1998, 261: 1-7

27 Lin Z, Wang X, Li Z, Ren SQ, Chen GN, Ying XT, Lin JM. Development of a sensitive, rapid, biotin-streptavidin based chemiluminescent enzyme immunoassay for human thyroid stimulating hor- mone. Talanta, 2008, 75: 965-972

28 Wang X, Lin JM, Ying X. Evaluation of carbohydrate antigen 50 in human serum using magnetic particle-based chemiluminescence enzyme immunoassay. Anal Chim Acta, 2007, 598: 261-267

29 Wang X, Zhang QY, Li ZJ, Ying XT, Lin JM. Development of high-performance magnetic chemiluminescence enzyme immunoassay for $\alpha$-fetoprotein (AFP) in human serum. Clin Chim Acta, 2008, 393: 90-94

30 Ren S, Wang X, Lin Z, Li Z, Ying X, Chen G, Lin JM. Micro-plate chemiluminescence enzyme immunoassay for clinical determination of progesterone in human serum. J Chin Anal Chem, 2008, 36: 729-734

31 Zhao L, Lin JM. Development of a micro-plate magnetic chemiluminescence enzyme immunoassay, MMCLEIA for rapid- and highthroughput analysis of 17-estradiol in water samples. J Biotechnol, 2005, 118: 177-186

32 Marshall JD, Eveland WC, Smith CW. Superiority of fluorecent- antibody technic with a modification of its application. Proc Soc Exp Biol Med, 1958, 98: 898-900

33 Lewis VJ, Jones WL, Brooks JB, Cherry WB. Technical considerations in the preparation of fluorescent-antibody conjugates. Appl Microbiol, 1964, 12: 343-348

34 Xin TB, Wang X, Jin H, Che JH, Liang SX, Li ZJ, Lin JM. Determination of estradiol in human serum using magnetic particles-based chemiluminescence immunoassay. Chin J Anal Chem, 2008, 36: 1056-1060

35 Dungchai W, Siangproh W, Lin JM, Chailapakul O, Lin S, Ying X. Development of a sensitive micro-magnetic chemiluminescence enzyme immunoassay for the determination of carcinoembryonic antigen. Anal Bioanal Chem, 2007, 387: 1965-1971 